\title{
A Red Fluorescence Two-photon Absorption Probe for Sensitive Imaging of Live Mitochondria
}

\author{
Makoto Tominaga, ${ }^{1}$ Shuhei Mochida, ${ }^{2}$ Hiroyuki Sugihara, ${ }^{2}$ Koichiro Satomi, ${ }^{1}$ Hiroki Moritomo, ${ }^{1}$ \\ Akinari Fuji, ${ }^{1}$ Arina Tomoyuki, ${ }^{3}$ Yasutaka Suzuki, ${ }^{* 1}$ and Jun Kawamata ${ }^{1}$ \\ ${ }^{1}$ Graduate School of Medicine, Yamaguchi University, 1677-1 Yoshida, Yamaguchi 753-8512 \\ ${ }^{2}$ Graduate School of Science and Engineering, Yamaguchi University, 1677-1 Yoshida, Yamaguchi 753-8512 \\ ${ }^{3}$ Faculty of Science, Yamaguchi University, 1677-1 Yoshida, Yamaguchi 753-8512
}

(E-mail: ysuzuki@yamaguchi-u.ac.jp)

We synthesized two-photon absorption probes based on naphthalene and anthracene: 1,1'-dimethyl-4,4'-(2,6-naphthylenedi-2,1-ethenediyl)dipyridinium diiodide (NP) and 1,1'-dimethyl-4,4'-(2,6-anthrylenedi-2,1-ethenediyl)dipyridinium diiodide (AC). These two probes were successfully accumulated in mitochondria with different fluorescence colors: yellow (NP) and red (AC). Two-photon absorption cross sections $\left(\sigma^{(2)}\right)$ were 712 and $685 \mathrm{GM}\left(1 \mathrm{GM}=10^{-50} \mathrm{~cm}^{4} \mathrm{~s}_{\text {photon }}{ }^{-1}\right.$ molecule $\left.^{-1}\right)$, respectively. These values are nine times larger than the $\sigma^{(2)}$ of the commercially available mitochondria-selective probe with efficient two-photon absorption property (rhodamine 123).

Two-photon fluorescence microscopy (TPFM) is known as an advanced technology for three-dimensional (3D) imaging of tissues and organs. ${ }^{1}$ This technique enables $3 \mathrm{D}$ imaging of blood flow, ${ }^{2}$ neural network, ${ }^{3}$ neutrophil movement, ${ }^{4}$ local oxygen concentration in the bone, ${ }^{5}$ and so forth. In a typical TPFM, the excitation wavelength is ca. $800 \mathrm{~nm}$ and the observed fluorescence is between 400 to $550 \mathrm{~nm}$. Transparency for biological tissues in the wavelength region from 400 to $550 \mathrm{~nm}$ is insufficient. Therefore, imaging at a point deeper than $1 \mathrm{~mm}$ cannot be achieved using the conventional TPFM probe. In contrast, a wavelength region from 600 to $1300 \mathrm{~nm}$ is known as the "tissue optical window". 6 Biological materials usually have low absorption in this window, so that light penetrates deeper inside the tissue. By employing the probe that can be both excited and emitted in the tissue optical window, deeper observation of tissue and organs by TPFM can be achieved.

We have developed an efficient two-photon fluorescence probe: 1,1'-dimethyl-4,4'-(9,9'-diethyl-2,7-fluorenediyl-2,1-ethenediyl)dipyridinium diperchlorate (FLW, Figure 1a). This probe

(a)

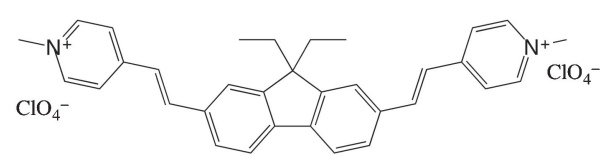

(b)

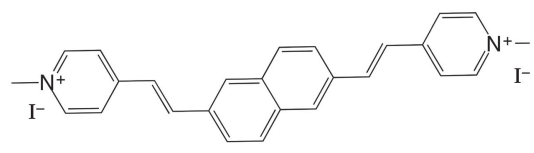

(c)

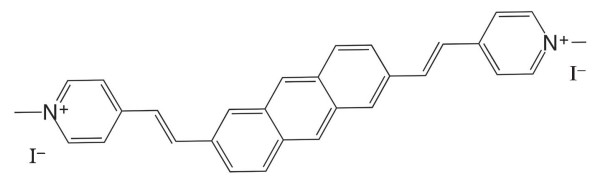

Figure 1. Chemical structure of FLW (a), NP (b), and AC (c). comprises a fluorene core and pyridinium groups. ${ }^{7}$ The cationic pyridinium moiety was designed to adsorb onto the negatively charged mitochondrial membrane through electrostatic interaction. The FLW showed a large two-photon absorption (TPA) cross section $\left(\sigma^{(2)}\right)$ with $750 \mathrm{GM} \quad\left(1 \mathrm{GM}=10^{-50}\right.$ $\mathrm{cm}^{4}$ s photon $^{-1}$ molecule ${ }^{-1}$ ) at $730 \mathrm{~nm}$. This value is larger than those of typical mitochondria-selective probes, for example, green fluorescent protein (GFP; $8 \mathrm{GM})^{8}$ and rhodamine 123 (80 GM). ${ }^{9}$ Although efficient TPA compounds are usually insoluble in the aqueous medium because of their hydrophobic character, the two cationic pyridinium moieties of FLW contribute to the increase of water solubility. FLW was soluble in water at a concentration on the order of $10^{-4} \mathrm{~mol} \mathrm{~L}^{-1}$ and stained cells without using any organic solvent. This advantage allowed longtime imaging of live cells for more than $24 \mathrm{~h}$ because toxicity due to organic solvents was not a concern. However, the fluorescence maximum of FLW was at $545 \mathrm{~nm}$, which is not in the range of the tissue optical window as well as conventional TPFM probes.

In this study, we aimed to develop our molecular design for a two-photon fluorescence probe that exhibits both excitation and fluorescence in the tissue optical window. Polycyclic aromatic hydrocarbons are known as efficient fluorophores. Fluorescence wavelengths of these fluorophores are reported to be longer than that of fluorene. ${ }^{10}$ Actually, the fluorescence wavelengths corresponding to the largest energy gap of fluorene, naphthalene, and anthracene are 300,311 , and $382 \mathrm{~nm}$, respectively. ${ }^{10}$ Therefore, we expected that fluorescence wavelengths of the analogs of FLW bearing the naphthalene or anthracene moiety instead of the fluorene moiety are longer than that of FLW. Here, we designed and synthesized two compounds: the naphthalene derivative 1,1'-dimethyl-4,4'(2,6-naphthylenedi-2,1-ethenediyl)dipyridinium diiodide (NP) (Figure $1 \mathrm{~b}$ ) and the anthracene derivative 1,1'-dimethyl-4,4'(2,6-anthrylenedi-2,1-ethenediyl)dipyridinium diiodide (AC) (Figure 1c). The synthetic procedures for both NP and AC are described in Supporting Information.

Absorption and fluorescence spectra of FLW, NP, and AC are shown in Figure 2. The absorption peak of FLW was observed at $420 \mathrm{~nm}$. The wavelength of the absorption peak of NP was observed at $424 \mathrm{~nm}$ and shoulders were observed at 381 and $410 \mathrm{~nm}$. Absorption peaks of AC were observed at 338, 356, 391,413 , and $477 \mathrm{~nm}$. The cutoff wavelengths of the absorption bands of FLW, NP, and AC were 480, 470, and $520 \mathrm{~nm}$, respectively. The cutoff wavelength of $\mathrm{AC}$ was observed at the longest wavelength among three compounds. Contrary to expectation, the cutoff wavelength of NP was shorter than that of FLW. Absorption peaks of nonsubstituted fluorene and naphthalene were reported at 206-301 and 221-320 nm, respectively, showing a longer wavelength of the absorption band of 


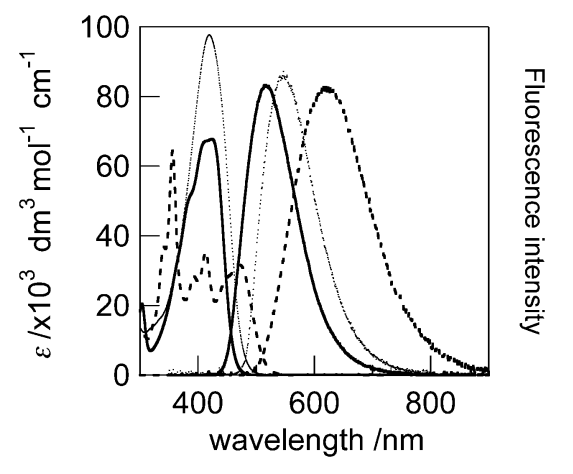

Figure 2. Absorption and fluorescence spectra of FLW (dotted line), NP (solid line), and AC (dashed line) in dimethyl sulfoxide.

naphthalene than of fluorene. ${ }^{11}$ However, the absorption band of NP was shorter than that of FLW. In FLW, NP, and AC, terminal pyridinium groups and central aromatic rings are categorized as electron acceptor (A) and electron donor (D) groups, respectively. The Hammett's substituent constant $\left(\sigma_{\mathrm{p}}\right)$ value, which represents electron donation ability, of the 2-fluorenyl and 2-naphthyl groups are -0.49 and -0.135 , respectively. ${ }^{12}$ Therefore, the intramolecular charge transfer of FLW would be larger than that of NP. This is the most probable reason for the absorption wavelength of NP being shorter than that of FLW. On the other hand, the absorption peaks of nonsubstituted anthracene were reported at $252-375 \mathrm{~nm}$; the wavelength of the absorption band of nonsubstituted anthracene was longer than that of nonsubstituted fluorene or naphthalene. The wavelength of the absorption band of $\mathrm{AC}$ was the longest among the three compounds, as expected.

As can be seen from Figure 2, the wavelength of the fluorescence maximum of FLW, NP, and AC were 542, 516, and $640 \mathrm{~nm}$, respectively. The fluorescence maximum of FLW was at a longer wavelength than that of NP, reflecting a longer cutoff wavelength of the absorption band. The fluorescence maximum wavelength of $\mathrm{AC}$ was the longest among the three compounds and was within the tissue optical window. The fluorescence quantum yields of FLW, NP, and AC in dimethyl sulfoxide were $0.44,0.36$, and 0.66 , respectively, whereas the values in water were $0.33,0.31$, and 0.08 , respectively. For aqueous solutions, fluorescence quantum yields of compounds exhibiting fluorescence at a longer wavelength region tend to be low. ${ }^{13}$ Thus, the decrease of the quantum yield of AC in water compared to that in an organic solvent should be considered to be a typical behavior of a fluorescence dye emitting in a longer wavelength region. The fluorescence quantum yield of $\mathrm{AC}$ in water was comparable to that of other red-fluorescence probes in water and thus, the fluorescence property of $\mathrm{AC}$ is thought to be sufficient for using it as a bio-imaging probe.

The $\sigma^{(2)}$ values of the compounds treated in this study were estimated by the open aperture Z-scan technique. ${ }^{14}$ The TPA spectra of FLW, NP, and AC are shown in Figures $3 \mathrm{a}-3 \mathrm{c}$. The maximum values of $\sigma^{(2)}$ of FLW, NP, and AC were $740 \mathrm{GM}$ at $780 \mathrm{~nm}, 712 \mathrm{GM}$ at $695 \mathrm{~nm}$, and $687 \mathrm{GM}$ at $703 \mathrm{~nm}$, respectively. The $\sigma^{(2)}$ of FLW, NP, and AC were comparable to each other. Thus, NP and AC maintained the efficient $\sigma^{(2)}$ of FLW. Furthermore, TPA bands of all probes were observed within the
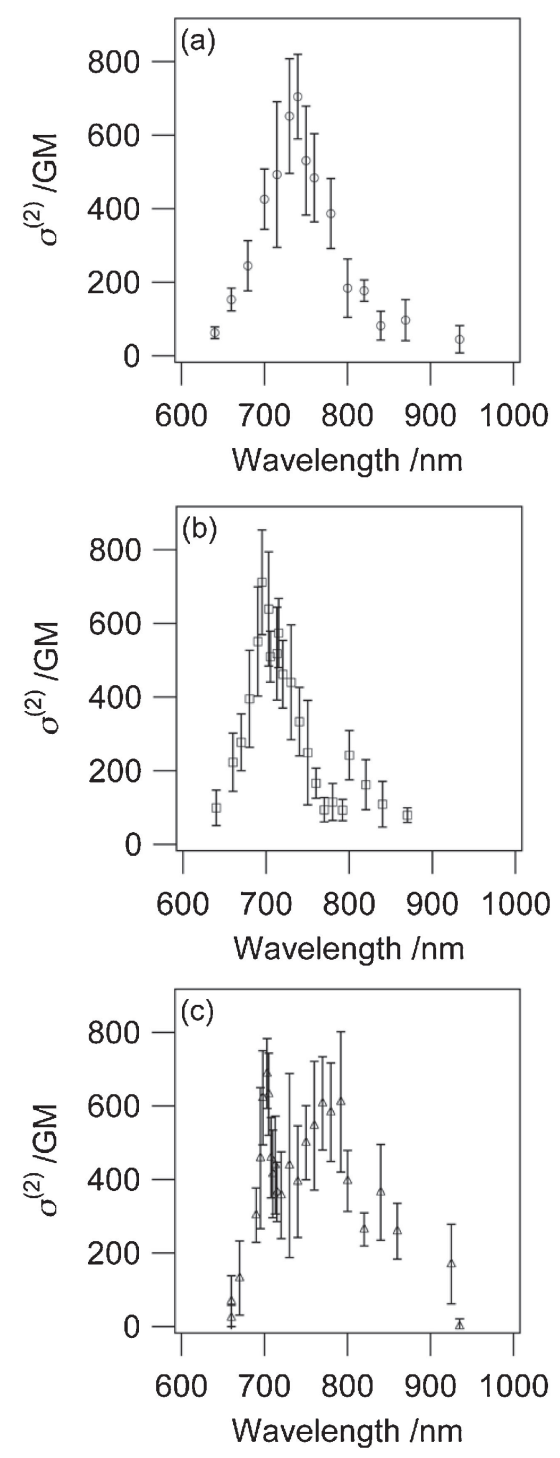

Figure 3. Two-photon absorption spectra of FLW (a), NP (b), and $\mathrm{AC}(\mathrm{c})$ in dimethyl sulfoxide.

tissue optical window. In the case of $\mathrm{AC}$, both excitation and fluorescence were within the tissue optical window.

To image living cells for a long time, use of organic solvents should be avoided in the staining procedure. Therefore, a probe, of which water solubility is higher than $10^{-7} \mathrm{~mol} \mathrm{~L}^{-1}$, is required. The water solubility of NP and AC was estimated to be $2 \times 10^{-4}$ and $6 \times 10^{-6} \mathrm{~mol} \mathrm{~L}^{-1}$, respectively. Although these probes have relatively larger $\pi$-electron systems, the water solubility was enough for introducing them into living cells as an aqueous solution.

The "one-photon" fluorescence images of HEK293 cells stained by NP and AC are shown in Figures $4 \mathrm{a}$ and $4 \mathrm{~b}$. The costaining fluorescence images of rhodamine 123, which is a mitochondria-selective probe, and FLW are shown in Figures 4c and $4 \mathrm{~d}$. The merged image of NP and rhodamine 123 is shown in Figure $4 \mathrm{e}$ and the merged image of AC and FLW is shown in Figure 4f. To avoid the overlap of the fluorescence color, a costaining experiment was conducted by using rhodamine 123 in 

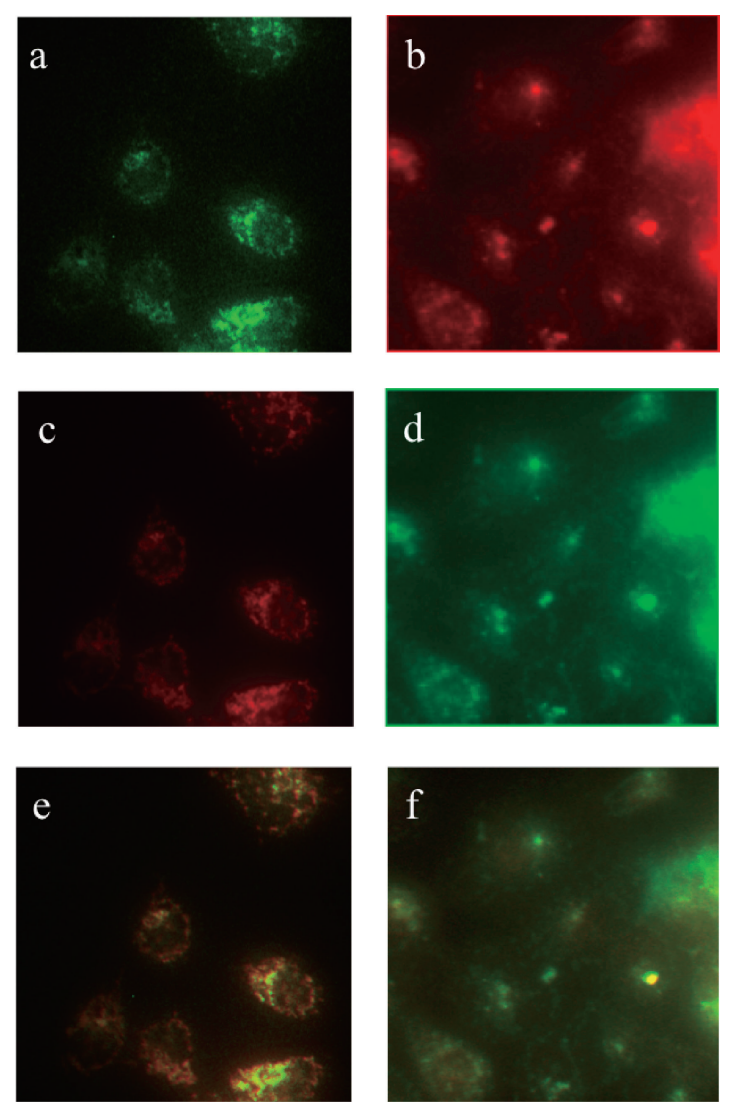

Figure 4. One-photon fluorescence microscope images of HEK293 cells. (a) shows cells stained with NP. (c) shows cells stained with rhodamine 123. (e) is a merged image of (a) and (c). (b) shows cells stained with AC. (d) shows cells stained with FLW. (f) is a merged image of (b) and (d).
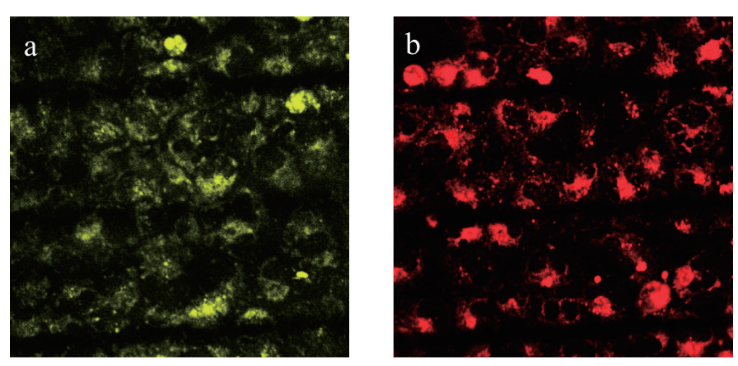

Figure 5. Two-photon fluorescence microscope images of HEK293 cells stained with NP (a) and AC (b). These images are obtained by $750 \mathrm{~nm}$ excitation and fluorescence were collected through a $500-600 \mathrm{~nm}$ band-pass filter.

the case of NP. The merged image (Figures 4e and 4f) revealed that the structures stained with NP and AC were very similar to those stained with rhodamine 123 and FLW. Thus, NP and AC were thought to accumulate in the mitochondria in HEK293 cells.

The TPFM images of HEK293 cells stained with NP and $\mathrm{AC}$ are shown in Figures $5 \mathrm{a}$ and $5 \mathrm{~b}$. Under this experimental condition, the averaged incident power of the excitation light source required to obtain TPFM images of $\mathrm{NP}, \mathrm{AC}$, and rhodamine 123 were 5,5 , and $50 \mathrm{~mW}$, respectively. Reflect to efficient TPA properties of NP and AC, sensitive observation can be accomplished by employing these probes.

In summary, we synthesized two novel water-soluble TPA probes based on naphthalene or anthracene: NP and AC. Although NP exhibited a shorter wavelength for fluorescence $(516 \mathrm{~nm})$ than the tissue optical window, greater TPA efficiency and water solubility were observed. In the case of $\mathrm{AC}$, we successfully controlled the fluorescence wavelength within the tissue optical window with enough water solubility, efficient TPA property, and mitochondria selectivity. Although introduction of polycyclic aromatic hydrocarbons much larger than anthracene to the present molecular back-bone would result in improved two-photon efficiency and a longer fluorescence wavelength, such structural modification would decrease water solubility. Therefore, AC is considered to be one of the most promising candidates for an efficient TPFM probe exhibiting both red fluorescence and sufficient water solubility.

Supporting Information is available electronically on J-STAGE.

\section{References}

1 W. Denk, J. H. Strickler, W. W. Webb, Science 1990, 248, 73.

2 J. Lecoq, A. Parpaleix, E. Roussakis, M. Ducros, Y. G. Houssen, S. A. Vinogradov, S. Charpak, Nat. Med. 2011, 17, 893.

3 R. Kawakami, K. Sawada, A. Sato, T. Hibi, Y. Kozawa, S. Sato, H. Yokoyama, T. Nemoto, Sci. Rep. 2013, 3, Article number 1014 .

4 T. Lämmermann, P. V. Afonso, B. R. Angermann, J. M. Wang, W. Kastenmüller, C. A. Parent, R. N. Germain, Nature 2013, 498, 371.

5 J. A. Spencer, F. Ferraro, E. Roussakis, A. Klein, J. Wu, J. M. Runnels, W. Zaher, L. J. Mortensen, C. Alt, R. Turcotte, R. Yusuf, D. Côté, S. A. Vinogradov, D. T. Scadden, C. P. Lin, Nature 2014, 508, 269.

6 R. Weissleder, Nat. Biotechnol. 2001, 19, 316.

7 S. Tani, K. Nakagawa, T. Honda, H. Saito, Y. Suzuki, J. Kawamata, M. Uchida, A. Sasaki, M. Kinjo, Curr. Pharm. Biotechnol. 2012, 13, 2649.

8 P. T. C. So, C. Y. Dong, B. R. Masters, K. M. Berland, Annu. Rev. Biomed. Eng. 2000, 2, 399.

9 R. Wolleschensky, T. Feurer, R. Sauerbrey, U. Simon, Appl. Phys. B: Lasers Opt. 1998, 67, 87.

10 Chemical Society of Japan, Kagaku Binran Kisohen II, Maruzen, pp. 759-760.

11 Chemical Society of Japan, Kagaku Binran Kisohen II, Maruzen, pp. 754-755.

12 Chemical Society of Japan, Kagaku Binran Kisohen II, Maruzen, p. 380.

13 a) M. C. Basheer, U. Santhosh, S. Alex, K. G. Thomas, C. H. Suresh, S. Das, Tetrahedron 2007, 63, 1617. b) L. Wang, J. Fan, X. Qiao, X. Peng, B. Dai, B. Wang, S. Sun, L. Zhang, Y. Zhang, J. Photochem. Photobiol., A 2010, 210, 168. c) B. Song, Q. Zhang, W.-H. Ma, X.-J. Peng, X.-M. Fu, B.-S. Wang, Dyes Pigm. 2009, 82, 396. 